\title{
Shape of stars and optical quality of the human eye
}

\author{
Rafael Navarro and M. Angeles Losada \\ Instituto de Optica Daza de Valdés, Consejo Superior de Investigaciones Cientificas, Serrano 121, \\ 28006 Madrid, Spain
}

Received December 1, 1995; revised manuscript received July 18, 1996; accepted September 17, 1996

\begin{abstract}
Star images are entoptic phenomena that most people can perceive when looking at bright point sources in darkness. Diffraction and/or ocular aberrations seem to be a plausible cause for the star patterns, but to our knowledge no objective recordings of retinal optical images showing these characteristic patterns have been reported before. We have projected a small Gaussian spot of light onto the retina and registered the aerial image formed externally through a fully dilated pupil \{one-and-a-half-pass method [J. Opt. Soc. Am. A 12, 2385 (1995)]\}. We have verified that, for fully dilated pupils $(>9 \mathrm{~mm})$, the blur caused by the finite size of the Gaussian spot is small. Consequently, these aerial images are a reasonably good approximation of the (inverted) optical point-spread function of the eye. These objectively recorded images displayed the distinctive radiating patterns of star images, which were compared with subjective patterns sketched out by the same observers. A strikingly close match was found between the objective and the subjective patterns of the same eyes. In addition, we computed the diffraction patterns produced by a simple schematic model of the suture lines of the anterior lens surface, also obtaining star-shaped images. These results support the commonly accepted hypothesis of a purely optical origin of subjective star images. (c) 1997 Optical Society of America [S0740-3232(97)00402-X]
\end{abstract}

\section{INTRODUCTION}

When looking at bright point sources in darkness, most people see characteristic ray patterns known as star images. The similarity of these subjective patterns to some of those typically found in optical diffraction phenomena suggests that star images could have an optical origin. The angular size of stars is generally very small compared with the resolution of the eye $(\sim 1$ arcmin). Thus stars are spatially coherent point objects, and the intensity distribution in the optical image is the point-spread function (PSF) of the image-forming optical system. On the other hand, rays appearing around bright points in optical images are often diffraction patterns resulting from the presence of sharp edges or defects in optical systems. Figure 1 shows examples of physical (optical) images of point sources and stars, as well as some drawings of subjective star images. The original picture was taken with a large telescope. ${ }^{1}$ Each star produces a PSF with a dim four-ray pattern, which is visible only for the brighter stars, T's. These diffraction patterns are produced by the structure supporting the secondary mirror of the telescope that breaks the pupil into four quadrants. (The ring appears as a result of the central occlusion.) This figure also contains examples of subjective star images. The eight-point star, $\mathrm{S}$, is a symbolic representation (detail of a sun clock of a Spanish gothic cathedral), and $\mathrm{C}$ is a more realistic hand drawing of the subjective retinal image of a bright point source viewed in darkness, ${ }^{2}$ which displays a series of tips, rays, and scattering halos.

If star images have a purely optical origin, ${ }^{2,3}$ then the optical PSF should display features similar to those of the star patterns reported subjectively by the same individuals. The subjective patterns (e.g., $\mathrm{C}$ and $\mathrm{S}$ ) have been known for a long time, but since the optical PSF had not been objectively recorded before, such an objectivesubjective comparison has not been done so far.

We have carried out this comparison using a recently developed objective method. ${ }^{4}$ A small Gaussian spot of light is projected onto the observer's retina. The eye's optical system forms the aerial image of that spot externally, which is captured with a CCD camera. This is the basis of the one-and-a-half-pass method, which gives a good approximation to the PSF of the eye provided that the Gaussian spot is small compared with the size of that PSF. ${ }^{4}$ Aerial images for several observers with fully dilated natural pupils $(\phi \geqslant 9 \mathrm{~mm})$ were recorded. Labels $\mathrm{MA}$ and RN in Fig. 1 correspond to some of these objectively recorded ocular PSF's, showing the distinctive radiating patterns of star images. The optical PSF's were compared with the subjective percepts by the same observers. Observers sketched out their subjective star images, which showed close agreement with the objective recordings, thus confirming their optical origin. On the other hand, the optical system of the eye contains structures, or optical defects, that could produce the diffraction patterns observed in star images. In particular we discuss, with the help of a computer simulation of a simple schematic optical model, how suture lines of the lens ${ }^{5}$ potentially could produce these kinds of radiating diffraction patterns (see $\mathrm{P}$ in Fig. 1).

\section{EXPERIMENTAL METHODS}

The basis of the one-and-a-half-pass method has been presented and discussed elsewhere. ${ }^{4}$ A narrow Gaussian pencil (waist $w_{0}=0.49 \mathrm{~mm}$ ) illuminates the retina, forming a diffraction-limited Gaussian $\operatorname{spot}^{6}$ (full width at half-height $\sim 1.8$ arcmin). The backscattered light then 


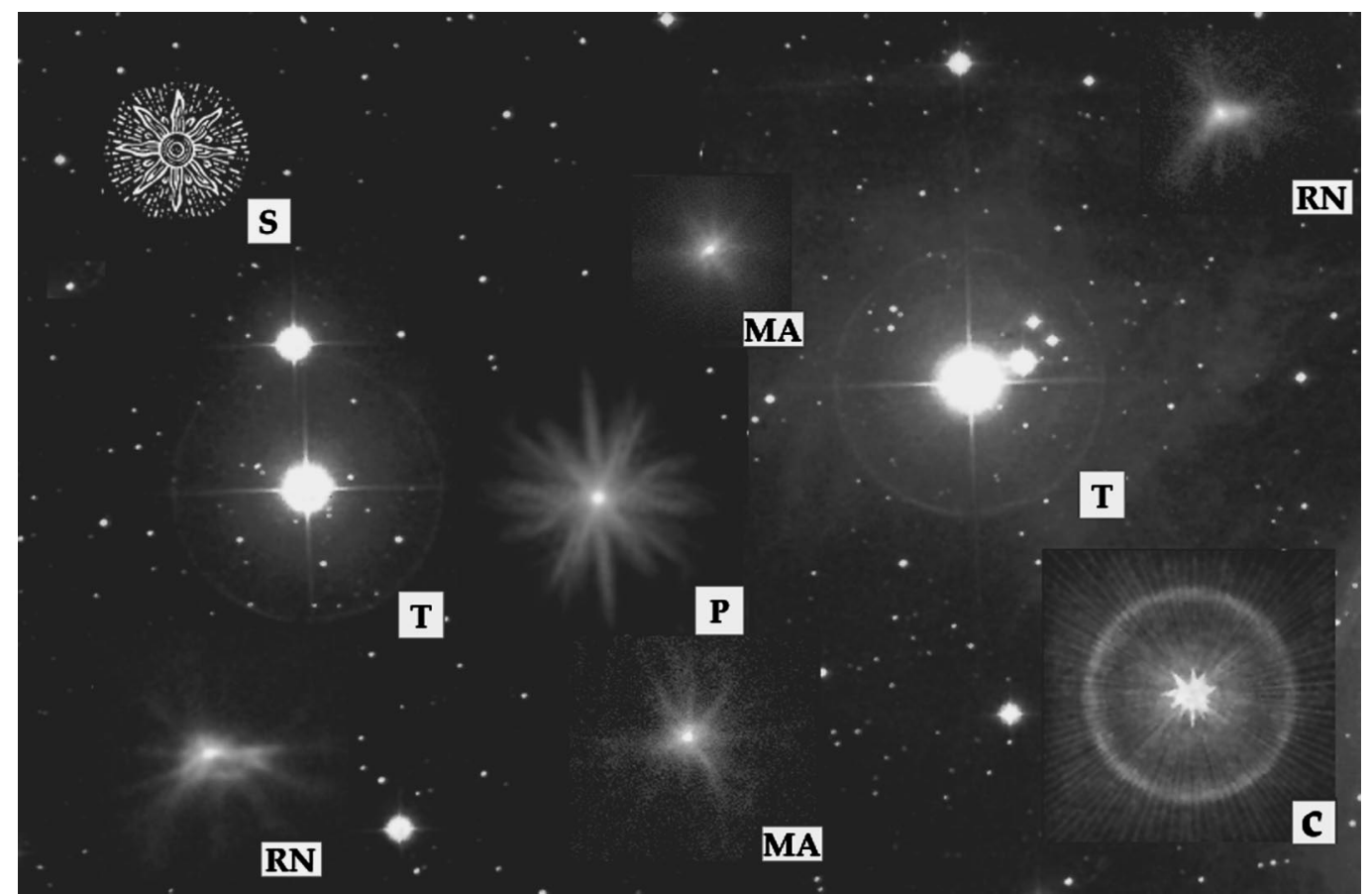

Fig. 1. "Virtual sky" artwork. Some stars in the original telescope image have been replaced by ocular images: T, original telescope images; S, symbolic representation; C, drawing of the subjective image of a bright point source ${ }^{3}$; $\mathrm{P}$, prediction by our computer simulation. MA and RN experimentally recorded retinal PSF's.

passes through the fully open pupil, forming an aerial image on a CCD camera (resolution 0.82 arcmin/pixel). This aerial image is the single-pass PSF cross correlated with the Gaussian spot. ${ }^{4}$ For a fully dilated pupil the PSF is much larger than the spot, and the aerial image is a slightly low-pass filtered version of the single-pass PSF. For this experiment we implemented a dual version of the setup, combining conventional symmetric-double-pass ${ }^{7}$ and one-and-a-half-pass configurations. The center of the observer's pupil was carefully aligned with the input beam both vertically and horizontally. The observer's pupil was fully dilated (final size slightly above $9 \mathrm{~mm}$ for all observers) by installation of two drops of tropicamide $1 \%$ during a 5 -min interval.

First, the observers viewed a point source through their dilated pupils with the standard symmetric-double-pass configuration. $^{7}$ (The point source was bright, but at a comfortable level.) They were instructed to look carefully at the point source and sketch out the pattern they perceived. For one naive observer, FR, we incorporated an illuminated ruler viewed through a beam splitter inserted in the path, at the same time as the point source. We asked this observer to use the ruler to measure the dimensions of his retinal image. All the drawings were made before recordings were taken in order to establish a blind comparison between objective and subjective patterns. Next we took four exposures of $5 \mathrm{~s}$ each (plus an additional recording of the background level) with this double-pass configuration to obtain the modulation transfer function (MTF) of the tested eye.

Then we switched to the one-and-a-half-pass configuration. We again asked the observers to pay attention to the new pattern generated by projecting the narrow beam onto the retina. All the observers reported that when the beam entered through the center of the pupil, they per- ceived a small rotationally symmetric ray-free point image. However, during pupil alignment, the narrow beam scanned lines across the observer's pupil in the horizontal and vertical directions, and sometimes the observer could eventually see some rays appearing and disappearing as we moved the observer's head. This suggests that the beam was crossing localized diffracting structures. Nevertheless, these rays were much fewer and much shorter than with the expanded beam, and when the narrow beam passed through the center of the pupil, no rays were visible at all.

We again took a series of four 5-s exposures plus an additional recording of the background level. The aerial image was computed as the average of the four exposures after the background was subtracted. The intensity of the beam was of the order of $10 \mu \mathrm{W}$, which is almost $2 \log$ units below standard safety limits. ${ }^{8}$

Five subjects (six eyes) with known prescriptions participated in the experiment. All tested eyes were normal except for one ambliopic (MR right eye):

- FR right eye (RE): sph. $=-0.5$ diopters (D), cyl. $=+2.5 \mathrm{D}$, axis $60^{\circ}$,

- MA RE: sph. $=-1.25 \mathrm{D}$, cyl. $=-0.5 \mathrm{D}, 85^{\circ}$,

- MR left eye (LE): sph. $=+0.25 \mathrm{D}$,

cyl. $=-0.25 \mathrm{D}, 105^{\circ}$;

$\mathrm{RE}: \quad$ sph. $=+9.75 \mathrm{D}, \mathrm{cyl} .=-1.5,40^{\circ}$,

- RN RE: sph. $=-0.75 \mathrm{D}$, cyl. $=-0.5 \mathrm{D}, 170^{\circ}$,

- UF RE: sph. $=+3.5 \mathrm{D}$, cyl. $=+0.25 \mathrm{D}, 10^{\circ}$.

( $-\mathrm{D}$ signifies myopic and $+\mathrm{D}$ hyperopic diopters.) First, all six eyes were tested with optical corrections. Observers FR, MR (left eye), and RN were also tested without correction. In addition, to study the effect of moderate amounts of defocusing on the ray patterns of star images, 
we made through-focus scans for observers MA and RN. For these observers we first compensated their refractive errors with trial lenses and took one series of recordings. Then \pm 0.75 spherical diopters were added, taking recordings of the aerial images in both cases. For throughfocus scans we took a series of eight 2 -s exposures, doubling pixel resolution to $0.41 \mathrm{arcmin} /$ pixel. The narrow Gaussian beam has a very low numerical aperture $(\sim 1 /$ 24) and produces an approximately diffraction-limited Gaussian, with a depth of focus (tolerance) of roughly \pm 3 D. Therefore the retinal spot will not be significantly affected by a $0.75 \mathrm{D}$ defocus.

\section{RESULTS}

Figure 2 shows four examples of the aerial images obtained. From now on, we shall represent intensity in a logarithmic scale to enhance the visibility of the lower values of star patterns. For this figure we have selected those aerial images that show more-visible ray patterns. Two of the displayed images, labeled MA and FR, were taken with refractive correction, and two, MR (LE), and $\mathrm{RN}$, were taken without correction. The images in these examples have similar sizes; about or slightly larger than $1^{\circ}$ of visual field. However, the variety of patterns found even with this limited sampling suggests that probably there are large differences among individuals in the geometry of the ray patterns.

Observers reported the subjectively perceived pattern to be very similar to the image recorded afterward and displayed by the computer. Nevertheless, the observers (except for the experimenters, MA and RN) sketched out their subjective star images, right before and under exactly the same conditions (refractive correction, alignment, etc.) as the objective recordings. Figure 3 displays three of these drawings, two made by observer FR (upper and center panels) and one by observer MR (LE) (lower panel). These subjective drawings show a remarkably close agreement with the corresponding objective experimental recordings of Figure 2. There is also agreement in orientation as a consequence of two facts: On the one hand, the visual field coordinates used by the observers for drawing the sketches have (inverted) opposed sign compared with that of the retinal coordinates. On the other hand, the lenses of the eye and of the setup form an inverted image of the retina on the CCD. Consequently, both sketches and recordings are inverted images of the retinal optical PSF, thus displaying the same orientation.
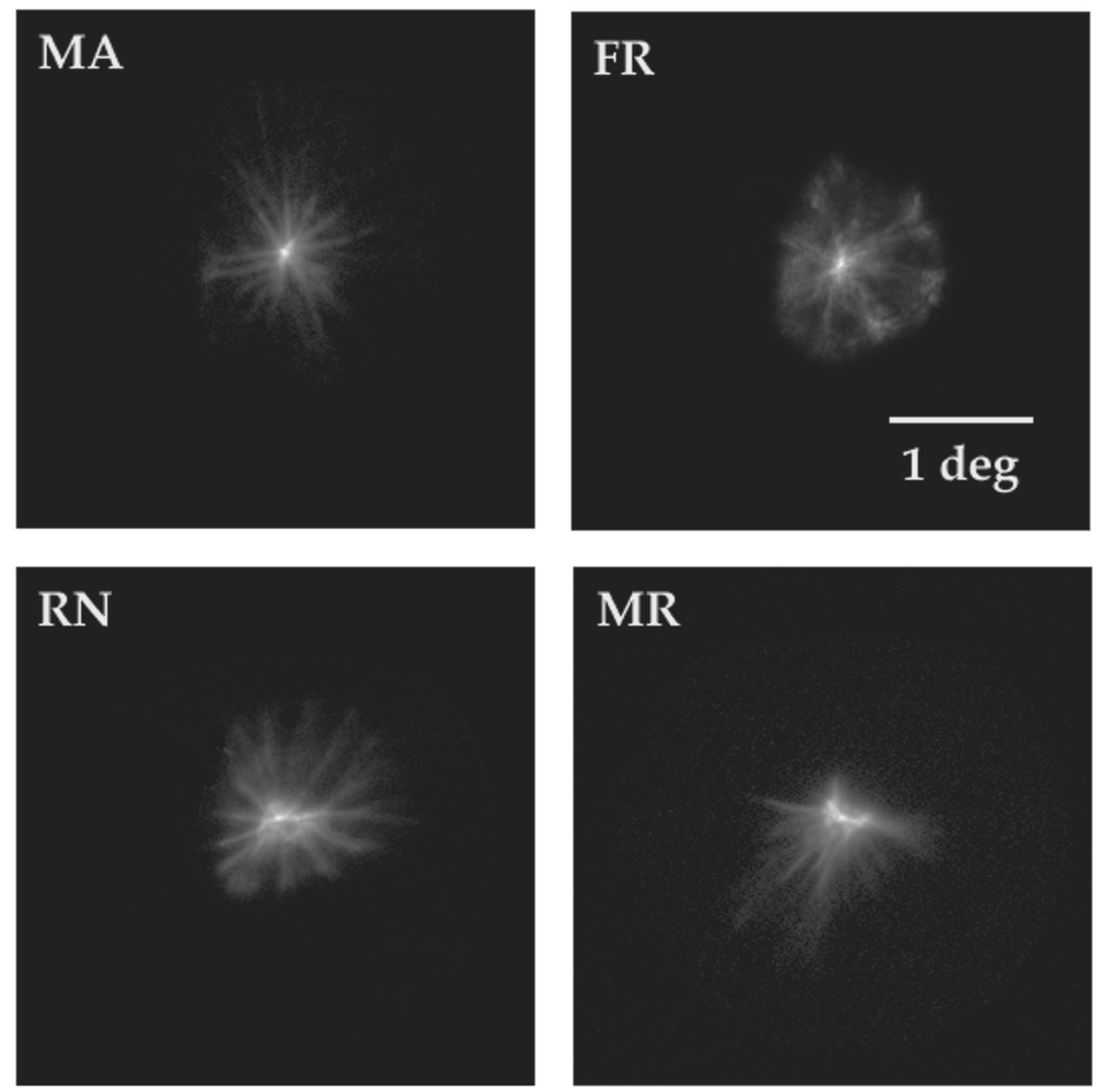

Fig. 2. One-and-a-half-pass aerial images for fully dilated pupils ( $>9 \mathrm{~mm}$ ). Low-intensity values have been enhanced by use of a logarithmic gray scale. These images show the characteristic visual patterns of star images, with sizes typically larger than $1^{\circ}$. 

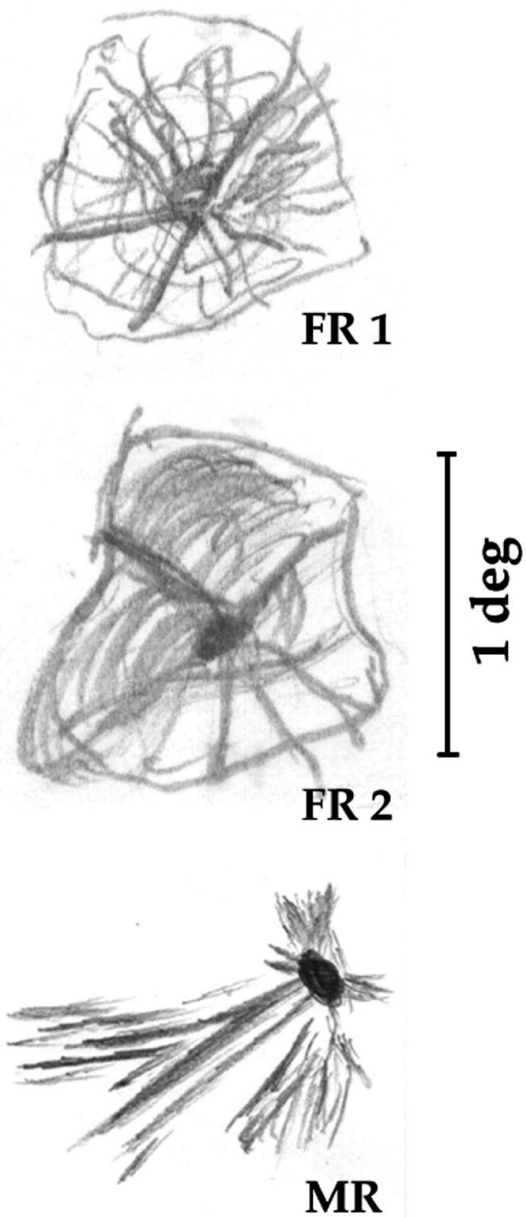

Fig. 3. Drawings made by observers FR and MR, who were trying to represent the subjective appearance of a bright point source when viewed with fully dilated pupils.

Furthermore, ob servers were asked to estimate the angular size of the subjective pattern, using some known reference viewed at the same time as the point source. In particular, for observer FR a ruler was viewed at the same time and same optical distance as the point object, permitting an accurate size measurement. The central panel of Fig. 3 includes the scale of the drawing as reported by this observer. As we can see, there is a close match between the angular sizes (in degrees) of the objective aerial image and the subjective star image for this observer.

These results suggest that our recorded aerial images are close to the perceived images of a bright point source. In Section 2 we mentioned that the aerial image is a good approximation of the eye's PSF only if the PSF is much greater than the spot that we project onto the retina. The case most likely to meet this condition is a fully dilated pupil, which will present large aberrations. If we compare the relative sizes of the recorded PSF's (greater than $1^{\circ}$ ) with the size of the Gaussian spot [1.9 $\operatorname{arcmin}$ (calibrated)], we see that the approximation is quite reasonable. (In optical engineering it is common to assume that an object is a point when its size is smaller than $1 / 5$ of the size of the PSF, which means an error of less than $2 \%$ in resolution.) Nevertheless, we tested further the influence of not having a true point object. For this purpose we compared (Fig. 4), the Fourier spectrum of the one-and-a-half-pass aerial image (filled circles), with the MTF of the same eye (open circles) for the same pupil. (The MTF has been estimated by the symmetric-doublepass method as the square root of the Fourier transform of the aerial image.) Both curves represent radial profiles (average over all orientations of the two-dimensional functions) for observer MA (refraction corrected). The Fourier transform of the theoretical Gaussian spot projected on the retina by the incident narrow beam has been also included (dotted curve) to illustrate its role as a lowpass filter. The retinal MTF (open circles) is far below the Fourier transform of the Gaussian spot as a result of the poor optical performance of the human eye for a fully open pupil $(>9 \mathrm{~mm})$. As a result, there is only a small, monotonically increasing offset between the Fourier spectrum of the one-and-a-half-pass aerial image and the eye's MTF. Therefore the one-and-a-half-pass aerial image, which is the cross correlation between the Gaussian spot and the retinal PSF, seems to be a close approximation to the latter (PSF) and is little affected by the former. This is true only for the particular combination of a narrow incident beam and a fully dilated pupil for the second pass.

The results of through-focus scans are illustrated in Fig. 5 for observer RN. In this figure the dioptric values correspond to the power of the lens used to defocus the retinal image; inserting negative lenses will produce hyperopic states and positive lenses myopic states. The top panel shows a typical retinal image found in moderate hyperopic refractive states, $-0.75 \mathrm{D}$, where ray patterns are hardly visible. In contrast, moderate myopia seems to favor the appearance and visibility of star patterns displaying longer rays (see bottom panel of Fig. 3). The same effect was found for subject MA. For these two observers the sign of defocus seems to induce an asymmetric change of star patterns near the plane of best focus. Adding a moderate amount of dioptric power with respect to the infocus refractive state seems to enlarge the ray pattern, whereas subtracting power seems to shrink the ray pat-

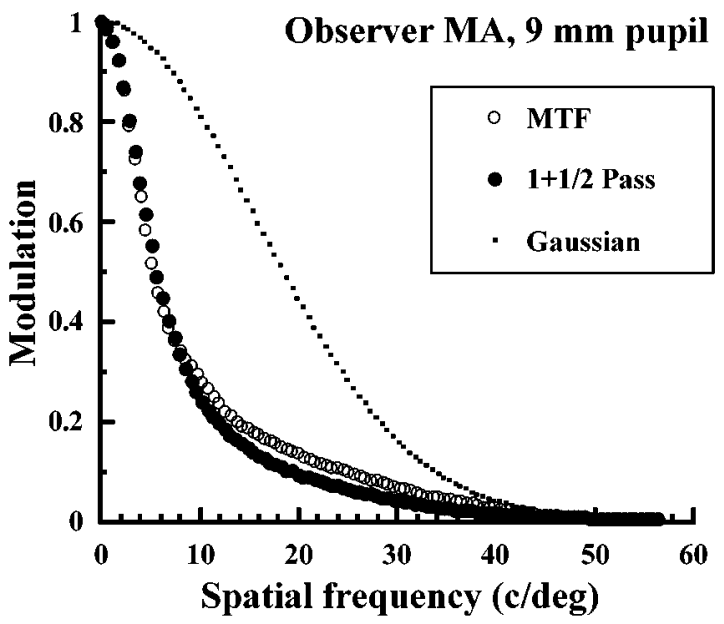

Fig. 4. Radial profiles of the Fourier transform of Fig. 3(a) (filled circles) and of the standard double-pass MTF for the same observer, MA (open circles). There is a small, monotonically increasing offset resulting from the Gaussian blur (dotted curve). 

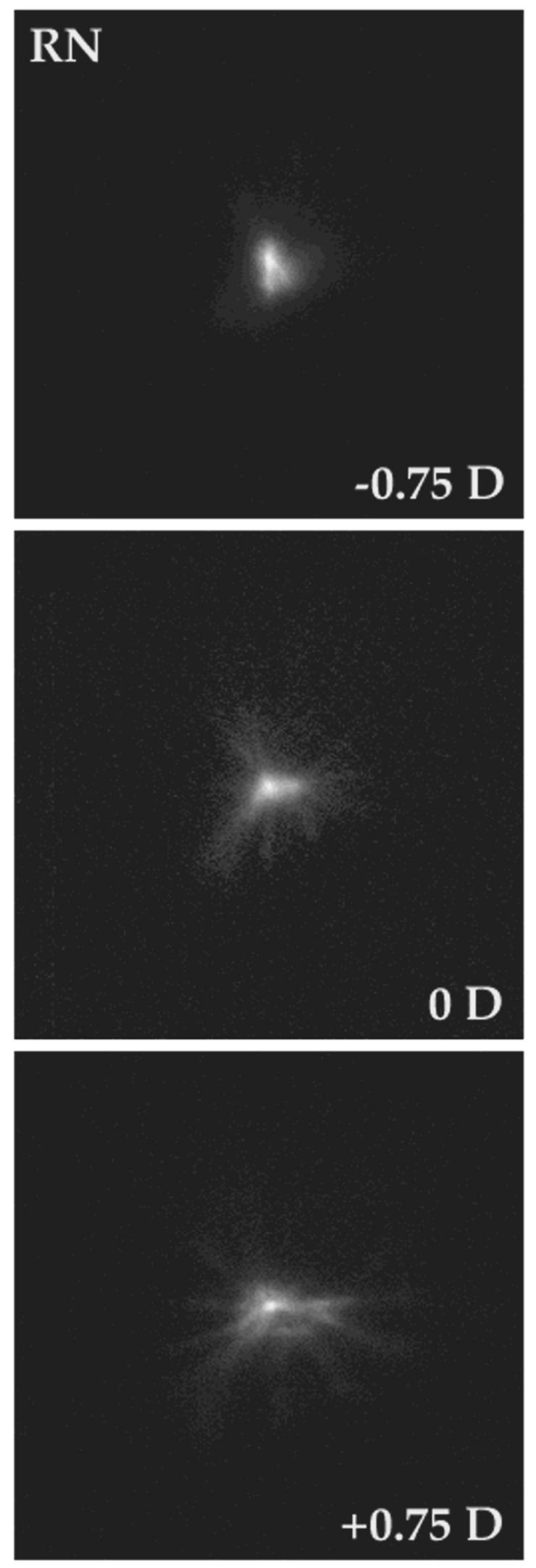

Fig. 5. Through-focus scan for observer RN (double magnification of Fig. 3). The ray pattern enlarges, enhancing its visibility, by positive (myopic) defocus, and it shrinks and fades with negative (hyperopic) values. tern. This would agree with a possible facilitation of the perception of star patterns by the phenomenon of night myopia, ${ }^{2}$ which could further enhance the perception of star patterns. For night vision the eye presents approximately 0.7 to 1.5 added diopters owing to accommodation lag plus a displacement of the best-focus plane resulting from larger aberrations. Then the global size and the visibility of the star pattern seem to depend on the refractive state.

\section{DISCUSSION}

As we stated in Section 1, star images have been known for a long time. They often appear in symbolic representations (see $\mathrm{S}$ in Fig. 1), and subjective drawings appear in classical $^{2}$ (C in Fig. 1) and modern textbooks. ${ }^{3} \mathrm{Al}-$ though related phenomena are commonly experienced during imaging of bright point sources with telescopes or photographic lenses, to our knowledge no objective recordings of retinal PSF's showing the distinctive pattern of star images had yet been reported. Here we report evidence supporting the assertion that star images are entoptic phenomena with an optical origin: We have compared subjective star images, perceived monocularly, with objective recordings of the optical PSF of the same eye. The objective results constitute, to our knowledge, the first in vivo external recordings of optical PSF's that show the distinctive pattern of star images. They are affected by a slightly low-pass filtering, but nevertheless, we have found the following:

- There is a close agreement between objective and subjective star-shaped patterns for individual eyes.

- For a narrow incident beam and a fully dilated pupil, the one-and-a-half-pass aerial image seems to be a reasonably good approximation of the PSF of the eye.

Since the projected Gaussian spot is small compared with the eye's PSF, the aerial image is a good approximation of the eye's optical PSF, although slightly low-pass filtered by cross correlation with the Gaussian spot. Observers confirmed perceiving the same pattern as the one recorded objectively when they were looking into an expanded beam that filled the dilated pupil of the same eye (with the standard double-pass configuration). The only difference, reported by experimenters when acting as observers, was perceiving somewhat sharper features as a result of a smoothing effect caused by the slight low-pass Gaussian filtering on the aerial images. Nevertheless, there are striking similarities between objective and subjective patterns, including size and orientation.

We found a large variety of patterns, even though we tested a limited number of observers (six eyes). Ray patterns appeared in observers with good refractive correction. The ray length increased in moderate myopic states, further enhancing the visibility of the ray patterns, whereas rays tended to vanish for moderate hyperopic refractive states. This tendency appeared consistently, although the sample of observers tested is too small to extrapolate a general rule. We have performed only a systematic (limited) through-focus scan for two observers, and further experiments and data analysis would be necessary to study this finding more thoroughly. A 
tentative, intuitive explanation for the effect of the sign of defocus on the length and visibility of the ray pattern could be the following: It has been argued that in night myopia the increment of spherical aberration with pupil size produces a displacement of the best image plane, inducing some "myopia." In other words, a defocus with opposite sign would partially compensate the effect of spherical aberration at the cost of displacing the image plane. Thus a defocus partially compensating the wave aberration (with the same order of magnitude and opposite sign) produces a better image than the in-focus case, whereas if both defocus and wave aberration have the same sign, the image is much worse. This produces an asymmetric effect of positive and negative moderate defocus. A similar explanation could perhaps apply to the phase of the diffracting structures or optical defects causing the ray pattern. Defocus of the same order and opposite sign could partially compensate for the peculiar defects. To test this and other issues further we carried out a computer simulation with a simple schematic model of the complex pupil function of the eye.

During observer alignment in the one-and-a-half-pass setup, we noticed that even the narrow Gaussian beam may produce some ray patterns when passing through some eccentric positions. If the observer is perfectly aligned and the beam passes through the center of the pupil, the subjective image is a ray-free round and clean (Gaussian) image. However, when we scan the pupil with a narrow beam as in a ray-tracing experiment, ${ }^{9}$ a limited number of rays eventually appear and disappear as we move the beam across the pupil. This suggests that we could be crossing some local diffracting structures or defects that are causing these ray patterns. The idea that star images are caused by fibrous structures in the lens is commonly accepted. ${ }^{3}$ In particular, suture lines of the lens ${ }^{5,10}$ (see Fig. 6) are local transparent structures produced by junctions of lens fibers, which potentially could cause diffraction patterns. Suture lines of the anterior surface are very close to the pupil and can be seen with a slit lamp, ${ }^{5,10}$ or coherent light. ${ }^{11}$ Anterior suture lines could cause a starlike diffraction pattern, since these transparent phase objects may distort the wave front, thus contributing to the wave aberration. In fact, phase objects often have a higher diffraction efficiency than amplitude objects.

To test this hypothesis, we computed the diffraction pattern (retinal PSF) of a crude schematic model of the complex pupil function, $P$, of the eye. We do not try to model individual results here, since this will require an accurate measurement not only of the wave aberration but also of the phase disturbances produced by the suture lines on the incident beam as it propagates in the forward direction. The modulus of the $P$ function is the pupil transmittance; we studied the influence of the StilesCrawford effect (SCE) by comparing the results of assuming a constant $9-\mathrm{mm}$ pupil (no SCE) or a Gaussian pupil intensity transmittance with a realistic width parameter, ${ }^{12} \rho=0.05$, inside the physical pupil. The phase of the pupil function was a standard Zernike polynomial (wave aberration) plus a phase map representing the suture lines. For this crude approximation we studied the effect of only some low-order aberrations: defocus and third-order astigmatism, coma, and spherical aberration. In the simulations we varied the values of each aberration, from zero to values above experimental data of the human eye, to study their separate and combined effects. For the suture lines there are no reliable experimental data available in the literature to define accurate phase maps. Although it is possible to take pictures of the suture lines by coherent or partially coherent illumination, it is very hard to determine the optical path difference that they produce. We have again adopted a rather simple approximation. We took a published schematic drawing of suture lines ${ }^{5}$ (Fig. 6) and transformed it into a phase map. The map was scaled to the size of the pupil (9 $\mathrm{mm}$ in diameter), and we assigned an arbitrarily constant phase lag to the suture lines. Since it is hard to obtain reliable estimates of this phase lag, we tested a number of values ranging from $\pi / 2, \pi$, and up to $10 \pi$. All these values provided basically the same diffraction pattern, changing only diffraction efficiency, i.e., the relative intensity of the ray pattern with respect to the central peak. These values yielded very similar and clearly visible diffraction patterns. A phase lag of $\pi$ provided the maximum intensity of the ray pattern, and this was the value used to obtain the results of Fig. 7. The resulting PSF's were obtained as the squared modulus of the Fourier transform of the complex pupil function. They were convolved with a Gaussian spot to simulate the one-anda-half-pass aerial images. Figure 7 compares two results (intensities are in log units): (a) shows the diffraction pattern obtained when only suture lines are considered (no aberrations and no SC apodization), and (b) shows the case in which all factors are considered. These diffraction patterns are strongly reminiscent of some of the classical star image shapes. The apodization caused by the SCE is clear: Rays are shorter and some even vanish as a result of the lower diffraction efficiency of those struc-

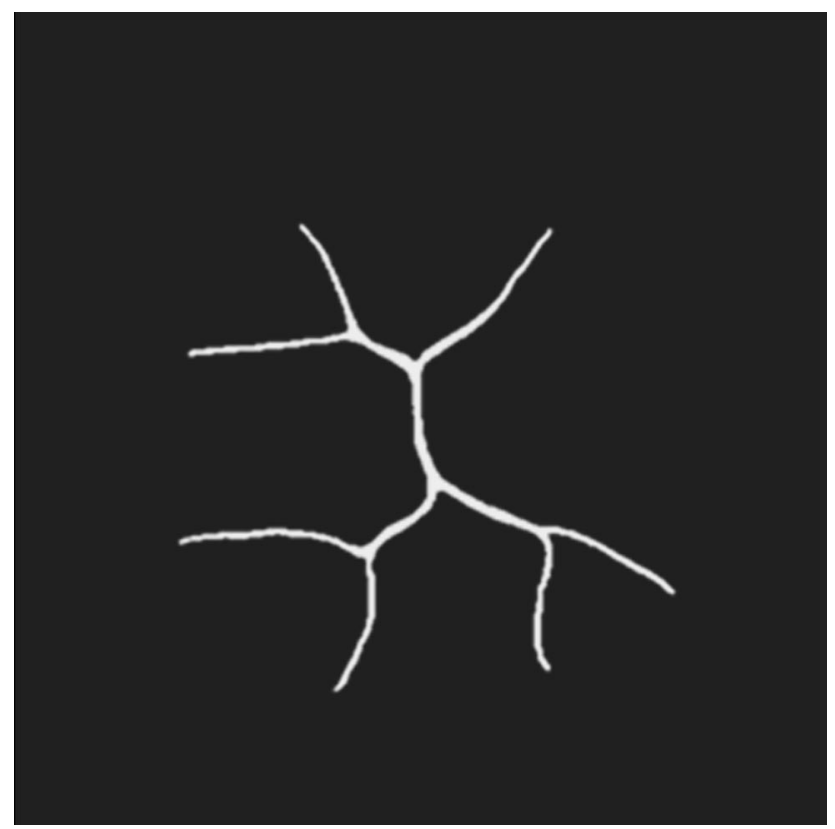

Fig. 6. Schematic drawing of the suture lines of the lens used as a phase map in the computer simulation (redrawn from Tripathi and Tripathi ${ }^{6}$ ). 

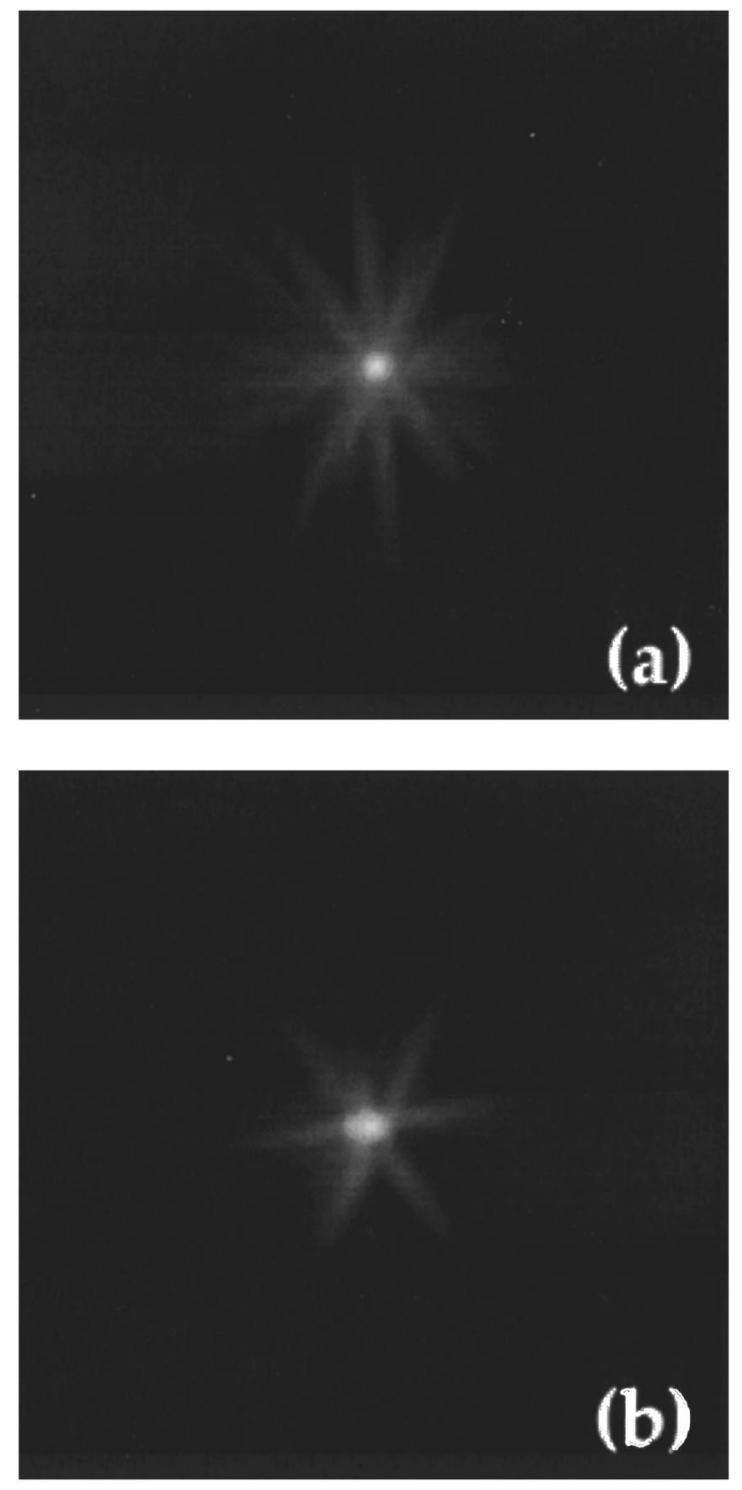

Fig. 7. Results of a computer simulation: (a) Diffraction pattern produced by the suture lines of Fig. 6. (b) Diffraction pattern obtained when including aberrations (defocus, astigmatism, coma, and spherical) and the Stiles-Crawford apodization. Intensities are given in a logarithmic scale.

tures located far from the pupil center. For this case we included $-2 \lambda$ (wavelengths) of defocus, $2 \lambda$ of astigmatism, $2 \lambda$ of coma, and $4 \lambda$ of spherical aberration. These values are arbitrary but of the same order of magnitude as those found in real eyes. ${ }^{13}$ These low-order aberrations seem to affect mainly the central region and not the ray pattern. In addition, the effect of the particular sign of defocus on the length of the rays was tested by computing the PSF's for different values of defocus ranging from $-10 \lambda$ to $+10 \lambda$ ( $1 \mathrm{D}$ of defocus is roughly equivalent to $6 \lambda$ ). We found the same basic asymmetric effect of moderate defocus that we obtained experimentally; i.e., the rays were shorter when the defocus had sign opposite from and magnitude similar to the suture lines, but the effect was much smaller than what we found in the experiments. A closer prediction would probably require not only a more accurate knowledge of the eye's pupil function, mainly of the optical path difference produced by the suture lines and higher-order aberrations, but also more experimental data in a larger population. Other potentially interesting studies could be done if we consider these patterns as a sort of signature of diffracting structures in the ocular media. In particular, we have seen that typical star images in normal eyes could perhaps be produced by suture lines of the lens. If this is true, then different optical defects, or even different refractive surgery techniques, should present different signatures in the tails of the PSF, as well. For example, eyes with clean intraocular lens implants should not present radiating patterns. On the contrary, radial keratotomy may show additional features.

\section{ACKNOWLEDGMENTS}

This research was supported by the Comisión Interministerial de Ciencia y Tecnología (Spain) under grant TIC940849. Our special thanks go to Francisco Romero, Maurice Rynders, and Ulrike Fempel for acting as observers and to Susana Marcos for help with computer simulations.

\section{REFERENCES}

1. J. Audouce and G. Israël, eds., The Cambridge Atlas of Astronomy, 2nd ed. (Cambridge U., Cambridge, U.K., 1988).

2. Y. Le Grand, Optique Physiologique. Tome III. L'Espace Visuel (Editions de la Revue d'Optique, Paris, 1956).

3. D. A. Palmer, "Entoptic Phenomena," in Visual Optics and Instrumentation, W. Charman, ed., Vol. 1 of Vision and Visual Dysfunction Series (J. R. Cronly-Dillon, General Editor) (MacMillan, London, 1991).

4. R. Navarro and M. A. Losada, "Phase transfer and pointspread function of the human eye determined by a new asymmetric double-pass method," J. Opt. Soc. Am. A 12, 2385-2392 (1995).

5. R. C. Tripathi and J. B Tripathi, "Morphology of the normal, aging, and cataractous human lens. I. Development and morphology of the adult and aging lens," Lens Res. 1\&2, 1-42 (1983).

6. B. E. A. Saleh and M. C. Teich, Fundamentals of Photonics (Wiley, New York, 1991), Chap. 3.

7. R. Navarro, M. Ferro, P. Artal, and I. Miranda, "Modulation transfer functions of eyes implanted with intraocular lenses," Appl. Opt. 32, 6359-6367 (1993).

8. D. H. Sliney and M. L. Wolbarsht, "Safety standards and measurement techniques for high intensity light sources," Vision Res. 20, 1133-1142 (1980).

9. R. Navarro and M. A. Losada, "Ray tracing in the living eye with a laser beam. Spot diagrams and aberrations," in Vision Science and Its Applications, Vol. 1 of 1996 OSA Technical Digest Series (Optical Society of America, Washington, D.C., 1996), pp. 230-233.

10. S. Duke Elder and K. C. Wybar, System of Ophthalmology, Vol. II of The Anatomy of the Visual System (Kimpton, London, 1961).

11. R. Navarro, J. A. Méndez-Morales, and J. Santamaría, “Optical quality of the eye lens surfaces from roughness and diffusion measurements," J. Opt. Soc. Am. A 3, 228-234 (1986)

12. R. A. Applegate and V. Lakshminarayanan, "Parameteric representation of Stiles-Crawford functions: normal variation of peak location and directionality," J. Opt. Soc. Am. A 10, 1611-1623 (1993).

13. H. C. Howland and B. Howland, "A subjective method for the measurement of monochromatic aberrations of the eye," J. Opt. Soc. Am. 67, 1508-1516 (1977). 